\title{
PRODUÇÃO DE MATERIAL DIDÁTICO EM ENSINO DE QUÍMICA NO BRASIL: UM ESTUDO A PARTIR DA ANÁLISE DAS LINHAS DE PESQUISA CAPES E CNPq
}

\author{
F. S. CUNHA ${ }^{1}$, S. K. G. OLIVEIRA ${ }^{1}$, J. P. D. ALVES ${ }^{1}$ e M. E. N. P. RIBEIRO ${ }^{2 *}$ \\ ${ }^{1}$ Instituto Federal do Rio Grande do Norte (IFRN) \\ ${ }^{2}$ Universidade Federal do Ceará (UFC) \\ elenirnp@yahoo.com.br*
}

Artigo submetido em setembro/2014 e aceito em junho/2015

DOI: $10.15628 /$ holos.2015.2423

\section{RESUMO}

Neste trabalho apresenta-se um estudo sobre as linhas e grupos de pesquisa em Produção de Material Didático em Ensino de Química no Brasil, uma área relativamente recente nos grupos de pesquisa nas Instituições de Ensino Superior (IES), entre o período de 2009 e 2014, visando saber quais IES tem esta linha e quais os seus objetivos, com base nos dados obtidos no Diretório dos Grupos de Pesquisa do CNPq e no relatório de Programa de Pós-Graduação que é enviado à CAPES. A pesquisa realizada constatou que o número de linhas de pesquisas na área de Produção de Material Didático em Ensino de Química é muito pequeno no nosso país, apesar do crescimento em pesquisas na área em questão, nos últimos anos. Ao todo, só existem quatorze linhas nessa área. Dessa forma, verifica-se a necessidade de haver mais linhas de pesquisa em Produção em Material Didático em Ensino de Química, para que haja mais auxílio ao professor em sala de aula.

PALAVRAS-CHAVE: Recursos Educacionais, Ensino de Química, Material didático.

\section{THE PRODUCTION OF DIDACTIC MATERIAL FOR SUPPORT CHEMISTRY TEACHING IN BRAZIL: A STUDY BASED ON A ANALYSIS MADE ON THE CAPES AND CNPq RESEARCH REPOSITORY}

\begin{abstract}
This paper presents a study on the Production of Teaching Materials in Chemistry Teaching's lines of research and research groups in Brazil - which is a recent area of study in Brazilian Higher Education Institutions (HEls) - in the period from 2009 to 2014. We intended to know which HEls have this line of research and what are their goals, based on a data obtained from the Directory of the CNPq Research Groups and from CAPES Graduate Program Report. The study found out that the
\end{abstract}

number of Production of Teaching Materials in Chemistry Teaching's lines of research is too small in our country, even with the growth in research in this area in the late years. There are only fourteen lines of research in this area. Based on this, there is a need for the creation of more lines of research of Production in Teaching Materials in Chemistry Teaching, so that there will be more material to the teachers of chemistry in the classroom.

KEYWORDS: Educational Resources, Chemistry Teaching, Didactic material. 


\section{INTRODUÇÃO}

A Pesquisa em Ensino em Química (PEQ) vem desenvolvendo atividades de Ensino, Pesquisa e Extensão que buscam somar ações para a melhoria da qualidade do ensino de Química, objetivando contribuir na reflexão e crítica por parte de pesquisadores que atuam nas Instituições de Ensino Superior (IES) no Brasil, expressando um novo olhar em relação aos grupos e linhas de pesquisa relacionados a esta área. É importante destacar que a PEQ é uma área recente de pesquisa com diferentes objetos de trabalhos das demais áreas de Química (as áreas mais clássicas como: Orgânica, Inorgânica e Físico-Química).

Nesse sentido, a PEQ vem promovendo ações que buscam reestruturar as bases curriculares e metodológicas, através de trabalhos com professores em sua realidade escolar. Tais ações ainda contribuem na aplicação do conhecimento químico por meio da busca de explicação da leitura dos fenômenos presentes, a partir de diversas situações presentes no cotidiano do aluno, sendo assim, o envolvimento com o professor é uma estratégia para minimizar problemas do ensino de Química, pois é ele o articulador do processo de ensinoaprendizagem em uma instituição.

Os questionamentos na área de PEQ, mesmo sendo uma área recente de estudo, desenvolvem-se, segundo Pérez, Silva e Nardi (2007), com maior ênfase de acordo com problemas de ensino e aprendizagem da Química, formação para a cidadania, produção e avaliação de materiais didáticos, currículos para o ensino de Química e formação de professores de Química.

Ainda que esta área de pesquisa seja nova em nosso país, há estudos nas universidades envolvendo o problema através de Grupos de Pesquisa, com objetivo de contribuir para melhoria do ensino de Química visando favorecer o desenvolvimento cognitivo dos estudantes e a formação de cidadãos conscientes.

De acordo com Borges-Andrade (2003), as linhas de pesquisas se configuram como um traço desenhado para determinar o rumo do que será investigado num dado contexto ou realidade, restringindo as fronteiras do campo específico do conhecimento em que deverá ser inserido o estudo. As linhas são usadas em programas de pós-graduação, em grupos de pesquisa e em curriculum vitae de pesquisadores, e nos contextos do CNPq e CAPES, sendo que nas linhas de pesquisa pode haver vários projetos.

Uma das linhas trabalhadas atualmente, de acordo com Oliveira, Miranda e Neto (2009), é a tecnologia digital para o ensino de Química que tem aparecido de forma considerável entre as linhas vigentes de pesquisa na área. O uso das tecnologias de informação e comunicação (TICs) é uma das formas de produção de material didático, que tem provocado mudanças nos setores da sociedade e em especial no ambiente escolar.

Nessa perspectiva, convém analisar os recursos usados nos processos de ensinoaprendizagem devendo ser adaptável, contextualizando o ensino de Química, levando em conta que nem sempre tais recursos são totalmente adequados para determinadas situações de ensino.

As linhas de pesquisa não são independentes umas das outras. Na verdade, se delineiam como um campo completo e de diversas interligações de notável interesse, uma vez que os 
resultados que derivam de suas investigações podem contribuir, em conjunto, para o melhoramento da qualidade da educação científica.

Dentre os diversos temas em linhas de pesquisa no Brasil, há uma preocupação crescente em relação à linha de pesquisa Produção de Material Didático em Ensino de Química. A visualização (recurso visual explorado pelo material didático) tem sido objeto de estudo recorrente, uma vez que pesquisadores da educação e professores de Química têm reconhecido a importância do assunto para o ensino dessa ciência.

Desta forma, o objetivo deste artigo é fornecer informações sobre as linhas de pesquisa em Produção de Material Didático em Ensino de Química nas IES do Brasil, nos últimos anos (2009-2014), e desta forma contribuir para o conhecimento de licenciandos e professores de Química de como e onde tem se desenvolvido pesquisa nessa área no Brasil.

\section{FUNDAMENTAÇÃO TEÓRICA}

Segundo Freitas (2009), os materiais e equipamentos didáticos - os quais são também conhecidos como "recursos" ou "tecnologias educacionais" - são "todo e qualquer recurso utilizado em um procedimento de ensino, visando à estimulação do aluno e à sua aproximação do conteúdo".

Existem muitos recursos nas escolas, tais como visuais, auditivos ou audiovisuais, ou seja, recursos que podem instigar/despertar o estudante por meio destas três formas de apropriação do externo. A visualização é de grande importância no ensino de Química, pois esta é uma ciência empírica. Desta forma, é necessário se aprender os modelos científicos já estabelecidos e aprender a desenvolver novos modelos de natureza tanto quantitativa quanto qualitativa por meio da visualização, conforme defendem Gilbert, Justi e Queiroz (2010).

Alguns estudos envolvendo a visualização mostram a necessidade de direcionar pesquisas sobre o tema, posto que há a necessidade de ampliar o entendimento sobre o assunto e suas implicações diretas e indiretas ao ensino de Química (TERUYA et al., 2013).

Ao fazer uso das representações visuais, é possível auxiliar os estudantes a aprenderem conceitos químicos, favorecendo a elaboração conceitual entre eles, que se tornam mais inseridos no processo de aprendizagem. Desta forma, segundo defende Vasconcelos e Leão (2010), a representação visual, quando incorporada à prática pedagógica, é uma ferramenta educacional.

Segundo Santos et al. (2010), os professores que constroem seus materiais ampliam seu conhecimento, pois constroem conhecimentos pela própria ação, e desta forma não são dependentes do livro didático. Assim encontram interstícios no conhecimento construído e aceitam o conhecimento, mas não de forma definitiva. Ainda de acordo com o autor, o professor é enriquecido nesse processo pelos conhecimentos que constrói, garantindo um ensino diferenciado aos alunos e propiciando uma reflexão mais ampla sobre o processo ensinoaprendizagem.

Para Freitas (2009), a presença dos materiais didáticos em si cumpre a função de estabelecer contato na comunicação entre professores e alunos, alterando desta forma o cotidiano de aulas apenas verbais. Como se sabe, cabe ao professor questionar sobre que 
materiais podem contribuir para reflexão do assunto a ser desenvolvido, considerando sempre a diversidade de linguagens, de formas de abordagem e de pontos de vista, assim o professor terá uma aula enriquecida quando escolhe o tipo e quando fazer uso do material didático em suas aulas.

A utilização de recursos didáticos como quadro branco (ou de giz) e pincéis, data show, jogos, passeios para pesquisa de campo e assim por diante, faz parte do processo de ensinoaprendizagem que é importante para que o aluno assimile o conteúdo trabalhado, desenvolvendo sua criatividade, coordenação motora e habilidade ao manusear objetivos diversos que poderão ser usados pelo professor na aplicação de suas aulas, servindo como mediadores nesse processo (SOUZA, 2007).

Nessa perspectiva, convém analisar os recursos usados nos processos de ensinoaprendizagem, devendo ser adaptáveis, contextualizando o ensino de Química, levando em conta que nem sempre tais recursos são totalmente adequados para determinadas situações de ensino.

Ao fazer uso das representações visuais, é possível auxiliar os estudantes a aprenderem conceitos químicos, favorecendo a elaboração conceitual entre eles, inserindo-os mais no processo de aprendizagem. Desta forma, a representação visual, quando incorporada à prática pedagógica, é uma ferramenta educacional que dá suporte como material didático em sala de aula.

$\mathrm{Na}$ tabela 1, encontra-se a relação de equipamentos didáticos existentes nas escolas brasileiras, que foram constituídos para atender os diversos componentes curriculares, em toda modalidade de ensino.

Tabela 1 - Classificação Brasileira dos Recursos Audiovisuais

\begin{tabular}{|c|c|c|}
\hline Recursos Visuais & Recursos Auditivos & Recursos audiovisuais \\
\hline Álbum seriado & Aparelho de som & Filmes \\
\hline Cartazes & Discos & Cispositivos e diafilmes com som \\
\hline Exposição & Fitas cassete & Televisão \\
\hline Fotografias & CDs & Computadoocassete \\
\hline Flanelógrafo & Rádio & Aparelho de DVD \\
\hline Gráficos & CD-ROM & Computador \\
\hline Gravuras & & \\
\hline Mapas & & \\
\hline Modelos & & \\
\hline Mural & & \\
\hline Museus & & \\
\hline Objetos & & \\
\hline Quadro de Giz & & \\
\hline Quadros & & \\
\hline Transparências & & \\
\hline
\end{tabular}

Fonte: Maria Rosângela Mello - CRTE Telêmaco Borba apud Freitas (2009).

A utilização de materiais usados no dia a dia em sala de aula, como exposição jornais, fotografias, livros, jogos e experiências permite que os alunos reconheçam aquele local como seu 
espaço, levando a ter afinidade exatamente porque devem circular por ela livremente tendo acesso a materiais, informações, regras de convivência e consequentemente isso atribuirá a construção do conhecimento (FREITAS, 2009).

Os materiais didáticos que circulam no meio escolar e servem como subsídio ou instrumento de apoio ao processo de ensino e aprendizagem têm despertado o interesse de pesquisadores de várias áreas de estudos, tornando-se um tema específico de pesquisa nas universidades (MELLO, 2010).

Pode-se citar como exemplo o artigo "A Utilização de Vídeos Didáticos nas Aulas de Química do Ensino Médio para Abordagem Histórica e Contextualizada do Tema Vidros", onde os autores Silva et al. (2012) descrevem uma experiência didática relacionada ao uso de vídeos como recurso didático para abordar o tema "vidros", numa perspectiva histórica e contextualizada no Ensino Médio. A atividade foi desenvolvida em três etapas: exibição e discussão dos vídeos e avaliação da sequência didática. O planejamento resultou na seleção de três vídeos para exibição e na elaboração de questionário avaliativo e o recurso vídeo foi considerado motivador

\section{PROCEDIMENTOS METODOLÓGICOS}

Esta pesquisa refere-se à análise dos grupos e linhas de pesquisa em Produção de Material Didático em Ensino de Química com o objetivo de identificar as tendências nos últimos seis anos no Brasil (2009 a 2014). Foi aplicada metodologia de natureza qualitativa e quantitativa. Como fontes para os trabalhos, foram utilizados: o levantamento no site do Diretório dos Grupos de Pesquisa do CNPq e o relatório de Programa de Pós-Graduação, que é enviado à CAPES, além de artigos referentes aos assuntos analisados. Considerando-se cada região no Brasil, descreveram-se quais IES possuem as linhas ou grupos de pesquisa em Produção em Material Didático, visualizando na área do Ensino de Química, que é uma área recente de pesquisa com diferentes objetos de trabalhos das demais áreas clássicas da Química (Química Inorgânica, Físico-Química, Química Analítica, Química Orgânica).

Para coleta de dados, adotou-se a quantificação das linhas e grupos de pesquisas nos diretórios CAPES e CNPq, procurando verificar os seus objetivos de estudos na PEQ, sobre Produção de Material Didático no Ensino de Química e em outras áreas, fazendo relação também com as áreas mais clássicas da Química, que tem um maior número de linhas de pesquisa voltado para pesquisa em si e não para o Ensino de Química.

\section{RESULTADOS E DISCUSSÃO}

Muitos cursos de pós-graduação com grupos e linhas de pesquisa em diversas áreas podem ser encontrados nas IES no país, subdivididos nas regiões Norte, Nordeste, Centro-Oeste, Sudeste, Sul, onde os sites da CAPES e CNPq fornecem informações detalhadas das pesquisas que são feitas em cada uma dessas instituições.

A CAPES, fundação do Ministério da Educação (MEC), desempenha papel fundamental na expansão e consolidação da pós-graduação stricto sensu (mestrado e doutorado) em todos os estados da Federação, onde suas atividades estão agrupadas nas linhas de ação, cada qual 
desenvolvendo um conjunto estruturado de programas: como a avaliação da pós-graduação stricto sensu; acesso e divulgação da produção científica; investimentos na formação de recursos de alto nível no país e exterior; promoção da cooperação científica internacional; indução e fomento da formação inicial e continuada de professores para a educação básica nos formatos presencial e à distância (CAPES, 2015).

O CNPq, agência do Ministério da Ciência, Tecnologia e Inovação (MCTI), tem como principais atribuições fomentar a pesquisa científica e tecnológica e incentivar a formação de pesquisadores brasileiros. Tem como missão, fomentar a Ciência, Tecnologia e Inovação e atuar na formulação de suas políticas, contribuindo para o avanço das fronteiras do conhecimento, o desenvolvimento sustentável e a soberania nacional (CNPq, 2015).

No entanto, essas agências financiam cursos de pós-graduação através de bolsas de manutenção para seus alunos e recursos para seu funcionamento, treinamento no país e no exterior, auxílio à pesquisa e apoio institucional, favorecendo um controle nos custeios e desenvolvimento científico e tecnológico do país. Uma característica da atuação dessas agências é a distribuição dos recursos e a análise de projetos nas linhas de pesquisas.

Para análise de dados levantados, fez-se uma divisão das linhas de pesquisa por região, primeiramente fazendo um levantamento do número de linhas de pesquisa nas áreas clássicas da Química, bem como das linhas relacionadas ao tema Produção de Material Didático em Ensino de Química nas IES.

Os dados das fontes pesquisadas mostram que as linhas de pesquisa nas áreas clássicas da Química são bem relevantes, conforme pode ser visualizado na Tabela 2.

Tabela 2 - Quantidade de linhas de pesquisa em pós-graduação nas IES, áreas clássicas da Química por Regiões no Brasil

\begin{tabular}{|c|c|c|c|c|c|}
\hline \multirow{2}{*}{ REGIÃO } & \multicolumn{4}{|c|}{ ÁREAS CLÁSSICAS DA QUIIMICA } & \multirow[b]{2}{*}{ TOTAL } \\
\hline & $\begin{array}{c}\text { QUÍMICA } \\
\text { INORGÂNICA }\end{array}$ & $\begin{array}{l}\text { FÍSICO- } \\
\text { QUÍMICA }\end{array}$ & $\begin{array}{c}\text { QUÍMICA } \\
\text { ANALÍTICA }\end{array}$ & $\begin{array}{c}\text { QUÍMICA } \\
\text { ORGÂNICA }\end{array}$ & \\
\hline Norte & 7 & 45 & 21 & 10 & 83 \\
\hline Nordeste & 31 & 141 & 92 & 61 & 325 \\
\hline Centro-Oeste & 5 & 54 & 29 & 19 & 107 \\
\hline Sudeste & 77 & 247 & 137 & 178 & 639 \\
\hline Sul & 31 & 118 & 70 & 64 & 283 \\
\hline Total & 151 & 605 & 349 & 332 & 1437 \\
\hline
\end{tabular}

Fonte: Própria (baseada em dados obtidos no sítio da CAPES e do CNPq).

Nas áreas mais específicas da Química (Inorgânica, Físico-Química, Analítica e Orgânica) existem diversas linhas de pesquisa, mas nenhum com objetivo de produção de material didático, e sim voltada para estudo experimental na área. Desde a colonização do Brasil começou haver divisão por estudos que, com o tempo, as mudanças tecnológicas e os avanços da pós-graduação do país, houve uma necessidade dessa divisão por áreas.

Para análise da pesquisa de acordo com os resultados obtidos, podemos observar a diferenças quantitativas das linhas por área, no qual se especifica a seguir. 
Química Inorgânica: É uma área que reúne um número relativamente pequeno em relação às outras áreas, com apenas cento e cinquenta e uma (151) linhas de pesquisa. Destaca-se mais na região Sudeste do país, tendo um número menor de linhas nas regiões do Norte e Centro-Oeste.

Físico-Química: Obtém um número maior de linhas nas IES, com seiscentos e cinco (605) linhas de pesquisa. É uma área que tem maior crescimento relativo no Brasil e maior diversidade se destacando especialmente na região Sudeste do país, sendo que o menor número de linhas de pesquisa se encontra nas regiões do Norte e Centro-Oeste.

Química Analítica: É uma área que reúne trezentas e quarenta e nove (349) linhas de pesquisa, perfil similar ao da Química Orgânica, mas em relação ao andamento da Físico-Química é lento. A maior concentração de pesquisa acadêmica se encontra na região Sudeste do país, sendo que o menor número se encontra nas regiões do Norte e Centro-Oeste.

Química Orgânica: É uma área que reúne trezentas e trinta e duas (332) linhas de pesquisa, ficando em terceiro lugar.

Neste contexto da análise das áreas predominantes da Química, podemos perceber a quantidade de linhas nessas áreas (Química Inorgânica, Físico-Química, Química Analítica, Química Orgânica) que se destacam com mil quatrocentas e trinta e sete (1437), onde a área que possui mais grupos e linhas no país é a área de Físico-Química apresentando um bom nível de crescimento na região Sudeste, e que segundo Santos (2002), um dos fatores de crescimento e de aumento foi a incorporação de moderna instrumentação, de projetos e a parcela dos laboratórios que se destaca no estado de São Paulo.

A Química é um importante instrumento para o desenvolvimento socioeconômico de qualquer país e, para o Brasil, tem contribuído significativamente no sentido do desenvolvimento de novos medicamentos, defensivos agrícolas, novos materiais para aplicações médica e eletrônica, dentre outras, cujos benefícios foram adquiridos pela sociedade (CORREIA; COSTA; FERREIRA, 2002). Dessa forma, observa-se que mesmo com tantos desafios a Química agrega grandes contribuições.

A SBQ tem contribuído nas diversas divisões clássicas da Química que tendem a desaparecer e gerar novas interfaces. A SBQ ganhou maturidade passando por dificuldades para fixar a ideia de uma Química de reconhecimento Internacional e que tem dado significativas contribuições à ciência no Brasil, abrindo espaços para novos desenvolvimentos que segundo Correia, Costa, Ferreira (2002 p. 80):

Para credenciar a Química no Brasil, é preciso não somente avançar na direção de novos conhecimentos científicos, mas também oferecer uma formação científica sólida aos nossos estudantes de graduação e pós-graduação, incluindo ainda a formação e a educação contínua dos professores do ensino médio, que de uma certa forma têm a importante incumbência de motivar os futuros cientistas deste país.

No entanto, para que haja interesse por parte dos estudantes desde o nível médio, precisa-se de metodologias motivadoras para uma melhor compreensão do conteúdo de 
Química. Nesse contexto, o professor é um mediador do conhecimento onde muitas vezes precisam de materiais que auxiliem nas suas aulas. Sabe-se que os materiais didáticos desempenham um papel que permite ainda ao aluno construir o seu próprio conhecimento.

Dentre desse contexto, buscou-se nos diretórios dos grupos no site da CAPES e CNPq a quantidade de linha em Produção de Material Didático no Ensino de Química. Observou-se que a quantidade dessa linha de pesquisa é relativamente pequena, pois só existem 14 linhas de pesquisa no país.

O gráfico da Figura 1 mostra a quantidade (em percentual) da Produção de Material Didático na área do Ensino de Química distribuída por região. Este gráfico deixa claro que a Região Sudeste tem posição de destaque no quadro geral da linha de pesquisa em Produção de Material didático, contando com um total de 9 linhas, enquanto a Região Sul não tem nenhuma linha. Este resultado pode ser reflexo da carência de mais pesquisadores na linha de pesquisa de recursos didáticos.

A Produção de Material Didático para o ensino de Química é uma área de pesquisa que necessita de atenção, haja vista que se configura como um campo novo, necessitando de um olhar diferenciado e um aumento expressivo no número de pesquisadores, mas para isso é preciso mais diálogos.

\section{PRODUÇÃO DE MATERIAL DIDÁTICO EM ENSINO DE QUÍMICA}

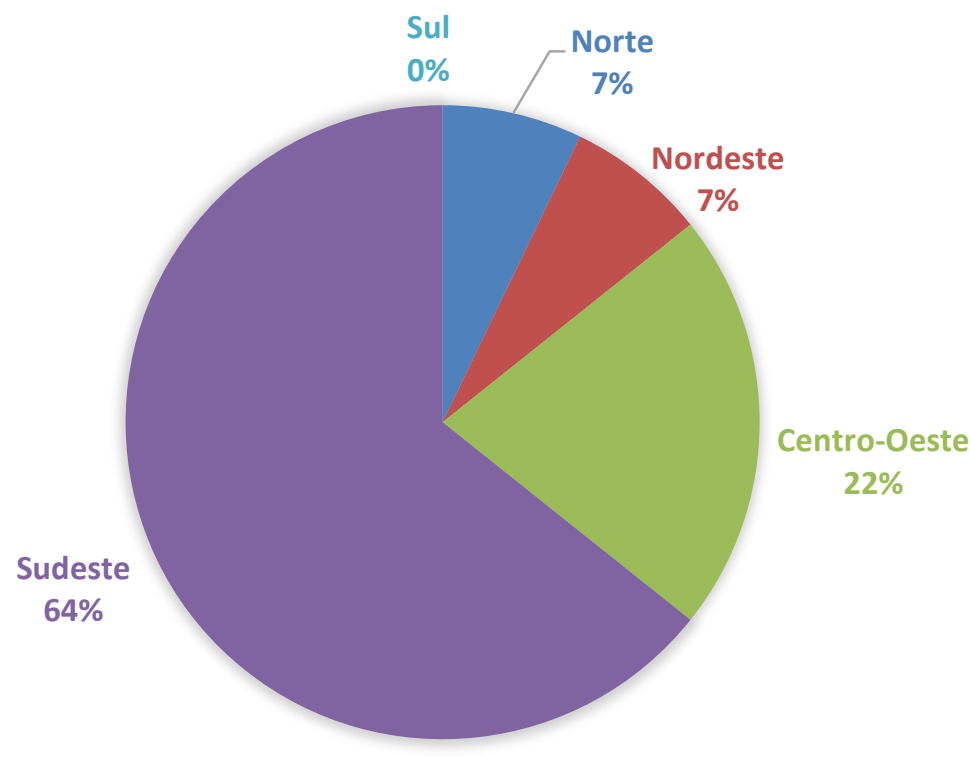

Figura 1 - Gráfico, em porcentagem, em Produção de Material Didático na área do Ensino de Química distribuída regionalmente. Fonte: Própria (baseada em dados obtidos no sítio da CAPES e do CNPq).

Nas tabelas a seguir (Tabelas 3 a 6) estão descritas o teor de pesquisa em Produção de Material Didático no Ensino de Química. 
Tabela 3 - Linha de pesquisa em Produção de Material Didático no Ensino de Química, na Região Norte

\begin{tabular}{|c|c|c|c|c|}
\hline Linha de Pesquisa & IES & $\begin{array}{c}\text { Última } \\
\text { Atualização }\end{array}$ & UF & Região \\
\hline $\begin{array}{c}\text { Experimentação e Produção de } \\
\text { Material Didático para o Ensino da } \\
\text { Química. }\end{array}$ & IFAP & Dezembro/2014 & AP & Norte \\
\hline
\end{tabular}

Fonte: CNPq.

Tabela 4 - Linha de pesquisa em Produção de Material Didático no Ensino de Química, na Região Nordeste

\begin{tabular}{|c|c|c|c|c|}
\hline Linha de Pesquisa & IES & $\begin{array}{c}\text { Última } \\
\text { Atualização }\end{array}$ & UF & Região \\
\hline Produção de material didático & UNEB & Dezembro/2014 & BA & Nordeste \\
\hline
\end{tabular}

Fonte: $\mathrm{CNPq}$

Tabela 5 - Linha de pesquisa em Produção de Material Didático no Ensino de Química, na Região Centro-Oeste

\begin{tabular}{|c|c|c|c|c|}
\hline Linha de Pesquisa & IES & $\begin{array}{c}\text { Última } \\
\text { Atualização }\end{array}$ & UF & Região \\
\hline Ensino-aprendizagem & UNB & Maio/2013 & DF & Centro-Oeste \\
\hline Ensino de Química & UFG & Maio/2013 & GO & Centro-Oeste \\
\hline Ensino e Tecnologias Educacionais & UFG & Maio/2013 & GO & Centro-Oeste \\
\hline
\end{tabular}

Fonte: CAPES.

Tabela 6 - Linha de pesquisa em Produção de Material Didático no Ensino de Química, na Região Sudeste

\begin{tabular}{|c|c|c|c|c|}
\hline Linha de Pesquisa & IES & $\begin{array}{c}\text { Última } \\
\text { Atualização }\end{array}$ & UF & Região \\
\hline $\begin{array}{c}\text { Ensino de química e ciências } \\
\text { Materiais Didáticos }\end{array}$ & UNIFRAN & Mario/2013 & SP & Sudeste \\
\hline $\begin{array}{c}\text { Desenvolvimento de experimentos } \\
\text { para o ensino de química }\end{array}$ & UFSCAR & Maio/2013 & SP & SP \\
\hline $\begin{array}{c}\text { Estratégias de ensino-aprendizagem } \\
\text { em química }\end{array}$ & UFSCAR & Maio/2013 & SP & Sudeste \\
\hline Ensino de Química & UNICAMP & Maio/2013 & SP & Sudeste \\
\hline $\begin{array}{c}\text { Preparação de Material Didático } \\
\text { para o Nível Superior }\end{array}$ & UNICAMP & Dezembro/2014 & SP & Sudeste \\
\hline $\begin{array}{c}\text { Preparação de Material Didático } \\
\text { para Abordagens Não Presenciais }\end{array}$ & UNICAMP & Dezembro/2014 & SP & Sudeste \\
\hline $\begin{array}{c}\text { Desenvolvimento e Utilização de } \\
\text { Animações no Ensino de Química }\end{array}$ & UNICAMP & Dezembro/2014 & SP & Sudeste \\
\hline $\begin{array}{c}\text { A Produção e Utilização de Crônicas } \\
\text { e Vídeos no Ensino de Química }\end{array}$ & UNICAMP & Maio/2013 & SP & Sudeste \\
\hline
\end{tabular}

Fonte: $\mathrm{CNPq} / \mathrm{CAPES}$.

Todas essas linhas de pesquisa têm como objetivo produzir material didático voltado para o Ensino de Química, no Ensino Médio, Ensino Superior e Ensino à distância, tem como propósito o ensino-aprendizagem, através de recursos didáticos nas instituições e escolas. 
Os equipamentos didáticos a serem trabalhados nas escolas para produção de materiais didáticos são inúmeros e variados. Uma descrição das diversas realidades distintas encontradas nas escolas brasileiras, assim como o uso de materiais e experimentos no ensino de Química, é uma forma de ensino capaz de facilitar o aprendizado, estreitando o contato entre o professor e aluno, podendo assim dinamizar a aula, facilitando a compreensão e incentivo para busca de mais conhecimento, contribuindo para sua formação como cidadão crítico e reflexivo.

A ideia do material didático como expressão de concepções de ensino e aprendizagem, significa um avanço em relação à concepção de material auxiliar. O material didático não é apenas um auxiliar, ele pode interferir de forma intensa na relação entre professor e aluno. Segundo Mello (2010), apesar das diferenças consideráveis ao nível da concepção do conteúdo e do modo como estes materiais são utilizados, reconhece-se atualmente que a qualidade e a intensidade do processo de interação entre os alunos e os materiais contribuem de modo decisivo para o resultado do processo de aprendizagem.

É necessário oferecer maior atenção às linhas de pesquisa em produção de material didático, especificamente na área do ensino de Química existentes no país, com a finalidade de avaliar e analisar quais os métodos e materiais trabalhados em sala de aula, para que venha ser algo motivador para os professores, principalmente os licenciados em estágio inicial da profissão, para que haja uma percepção no transmitir e mediar os conteúdos e conceitos da disciplina de Química de uma maneira mais integrada e contextualizada com suas vivências.

\section{CONCLUSÃO}

A linha de pesquisa em Produção de Material Didático em Ensino de Química é uma área que ainda precisa ser muito trabalhada, uma vez que é um campo novo nas IES do Brasil. Este trabalhou possibilitou uma visão das IES que têm se dedicado a esta linha de pesquisa e desta forma contribuir para o conhecimento do que está sendo desenvolvido no país. Porém, a prática docente precisa visualizar a importância e diversidade dos materiais didáticos, para que possa usufruir das possibilidades e melhorias nas metodologias. Posto que, os materiais, conforme destaca Freitas (2009), podem minimizar os efeitos de simples memorização, contribuindo para o desenvolvimento de ações de análise e síntese, generalização e abstração, a partir de elementos concretos, ampliando a experiência do estudante, facilitando o ensino-aprendizagem.

\section{AGRADECIMENTOS}

Os autores agradecem à PROPI-IFRN, COPEIN-IFRN/CN, PIBID/CAPES. Os autores ressaltam que este trabalho foi premiado em $1^{\circ}$ Lugar na Área de Educação na II Semana de Química IFRN, realizada de 02 a 04 de setembro de 2014 em Apodi/RN.

\section{REFERÊNCIAS BIBLIOGRÁFICAS}

1. BORGES-ANDRADE, J. E. Em busca do conceito de linha pesquisa. Revista Administração Contemporânea, Vol. 7, no 2, 2003. Disponível em: . Acesso em 10 set. 2013.

2. CAPES. Portal de Periódicos da Capes. Disponível me Acesso em: 03 jan. 2014. 
3. CNPq. Plataforma Lattes. Disponível em: Acesso em: 04 jan. 2014.

4. CORREIA, C. R. D.; COSTA, P. R.R.; FERREIA, V. F. Vinte e Cinco Anos de Reações, Estratégias e Metodologias em Química Orgânica. Química Nova. v.25, s.1, p. 74-81, 2002.

5. FREITAS, Os Equipamentos e materiais didáticos. Brasília: Universidade de Brasília, 2009. Centro de Educação a Distância. Disponível: Acesso em: 13 set. 2014.

6. GILBERT, J. K.; JUSTI, R.; QUEIROZ, A. S. Em The use of a model of modelling to develop visualization during the learning of ionic bonding; Tasar, M. F.; Cakmakci, G., eds.; PegemAkademi: Ankara, 2010.

7. MELLO, P.E.D. Materiais Didáticos para a Educação de Jovens e Adultos: História, Forma e Conteúdo. 2010. 254f. Tese (Doutorado - Programa de pós-graduação em Educação)Faculdade de Educação da Universidade de São Paulo. São Paulo: FEUSP, 2010.

8. OlIVEIRA, W. S.; MIRANDA, N. F.; NETO, J. M. M. Panorama da pesquisa em ensino de química no Brasil. 2009. Disponível em: . Acesso em: 10 set. 2013.

9. PÉREZ, L.F.M.; SILVA, C. S.; NARDI, R. Tendências na Pesquisa em Ensino de Química no Brasil e na Colômbia: Um estudo a partir da análise de publicações em revistas e anais de eventos. Anais do VI Encontro Nacional de Pesquisa em Educação em Ciências (VI ENPEC). 2007. Disponível em: . Acesso em: 13 set. 2014.

10. SANTOS, P. S. SBQ - 25 Anos: Físico-Química. Química Nova, v. 25, p. 31-33, 2002.

11. SANTOS, E. S.; HENRIQUE, H. C. R.; FERNANDES, A. M.; SILVA, R. M. G. Produção e Desenvolvimento de Materiais Didáticos Digitais para o Ensino de Química: Uma perspectiva formativa. XV Encontro Nacional de Ensino de Química (XV ENEQ), Brasília- DF, 2010.

12. Silva, J.L.; Silva, D.A.; Martini, C. Domingos, D.C.A.; Leal, P.G.; Beneditti Filho, E.; Fiorucci, A.R. A Utilização de Vídeos Didáticos nas Aulas de Química do Ensino Médio para Abordagem Histórica e Contextualizada do Tema Vidros. Química Nova na Escola, v. 34, n.4, p. 189 - 200, 2012.

13. TERUYA, L. C.; MARSON, G. A.; FERREIRA, C. R.; ARROIO, A. Visualização no ensino de química: apontamentos para a pesquisa e desenvolvimento de recursos educacionais. Química Nova, v.36, p. 561-569, 2013.

14. VASCONCELOS, F. C. G. C. LEÃO, M. B. C. A Utilização de Programas de Televisão como Recurso Didático e Aulas de Química. XV Encontro Nacional de Ensino de Química (XV ENEQ), Brasília- DF, 2010. 